When there is an injury the wound indicates the site for operative procedures. If there be no external guide, dependence must be placed upon localizing signs, providing they be sufficiently positive. Otherwise in suspicioue cases one is justified in opening the head over the region of the middle meningeal artery which, according to Treves, crosses the anterior and inferior angle of the parietal bone at a point one and one-half inches behind the external angular process of the frontal bone and one and three. quarter inches above the zygoma. The center-pin of the trephine should be inserted one and one-half inches behind the external angular process and one inch above the zygoma. Should the hole show no hemorrhage it may be enlarged upward and backward, or a second button may be removed just below the parietal eminence, this point being over the posterior branch of the artery.

The time of selection for operation is not later than eighteen tu twenty-four hours after the hemorrhage, and yet the surgeon should not hesitate to operate much later than this, since the clot may remain for months, as in the case reported by McBurney, of New York, in which he trephined four months after the injury and removed a clot which was still unabsorbed and causing trouble-securing a favorable result.

If the patient be unconscious no anesthetic is necessary. The method of opening the skull must depend upon the instruments at hand or the operator's choice between the trephine, the mallet and chisel, and the gouge and rongeurs. The clot when discovered should be removed by the fingers or forceps and the irrigator allowed to flow into the cavity for a minute or two, so that all remnants of biood may be washed out and any bleeding vessel uncovered. If a ruptured vessel be found in the dura, it must be ligated by catgut passed beneath it with a curved needle, and tied securely. If the main artery in the bony canal be the source of trouble, the bleeding may usually be controlled by punching with the sharp end of the handle of the bone forceps; in extraordinary cases it may be necessary to crush the bone upon the vessel by strong sequestrum forceps. Vessels in the pia and beneath, like the Sylvian, can be readily caught by passing a fine curved needle beneath, carrying a catgut ligature for tying. Not much force should be used in ligating vessels in this location for the reason that they rupture very easily under even moderate strain. When a general oozing is found, or hemorrhage from points not accessible, the only method to pursue is to pack with iodoform gauze, then close the scalp wound and apply a firm compress over all ; the tampon should be removed in thirty-six to forty-eight hours.

In conclusion, $\bar{I}$ give you this summary, under the heading of

\section{RULES FOR TREPIINING.}

When to Operate.-1. An injury to the head, followed after a little time by marked cerebral symptoms, imperatively demands trephining at the point of injury if known; if not, then over the middle meuingeal artery of the affected side.

2. In progressive or extradural hemorrhagecaused by a fall on the head or a blow from a dull instrument like a sand-bag-if symptoms of compression appear after many hours, or even days, there being no fracture or contusion as a guide, it is proper to open the skull over the middle meningeal artery; if no trouble be found there, then over the posterior branch.

3. Cases of "compression" or "concussion" without localizing symptoms or external evidence of injury should be subjected to exploration if there be no re. turn of consciousness within eighteen hours at the farthest; upon both sides if necessary.

4. Giddiness, headache and vomiting, with stupor and convulsions of one side, may be due to meningeal hemorrhage; exploration is permissible.

When not to Operate. -1 . When the heart's action is depressed, respiration irregular and swallowing difficult, the hemorrhage is probably subarachnoidean and operation can not be of benefit.

2. In cases dependent upon fracture, if blood be discharged from the mouth or ear there is basal injury and the justifiability of operation is questionable

\section{ORIGINAL ARTICLES.}

\section{SOME POINTS PERTAINING TO THE PRESENT PATHOLOGIC AND THERAPEUTIC STATUS OF DIPHTHERIA. BY I. N. LOVE, M.D. st. Lous, Mo.}

It will be admitted that until recently the management of diphtheria upon the part of the medical profession as well as many other diseases, has been empirical. Since the definite cause of this disease has seemed to be established, we have been able to work more intelligently in our treatment. It must not be forgotten, however, that able men in the profession do not yet accept the germ theory of disease. Among the number so great a man as Dr. Lawson Tait affirms his disbelief. He is of the opinion that these organisms abound and multiply under two conditions or states-decomposition and disease. He thinks their presence is incident to but not causative of disease. To illustrate his idea, he presents the homely illustration of the dead animal under the mid-summer sun. The prolific development of tissue destroyers in the form of maggots from microscopic parasites does not demonstrate to him that the maggots had anything to do with the death of the animal, but that the vitality of the latter, having been reduced to zero, the maggots, germs and spores utilize the opportunity for vital development upon their own part. He with others, believes that the various organisms operating under the conditions of disease and decomposition, attempt to act as scavengers for the removal of effete matter and perverted secretions and excretions rather than as the causes of morbid conditions. Discussing this subject before the Birmingham Midland branch of the British Medical Association last October, Mr. Tait threw hot shot against the germists by citing the difference of opinion among dermatologists regarding the parasitic origin of eczema; he quotes many of their best observers who believe that it is of neurotic origin and that microbes have nothing to do with it. He aptly says that if we can not settle upon the germ as being the cause of a disease so superficial, so exposed to view, so universal and so open to study as eczema, that there are few cases where it is capable of settlement. We can many of us sympathize with Tait when he says: "I entered my college career just as the grand sweep of the cellular pathology was careering over the medical world. Everybody was mad after a new cell. Special courses and special 
teachers were told off for cellular instruction. Our microscopes had got so far as to unriddle the facts of cellular construction, and therefore there must be a cellular pathology which was to explain everything. Two parties existed and they fought bitteriy, the only real and permanent effect being that the unhappy student had to meet two sets of examiners and to know two sets of answers to the same questions; the one to be given as the wind blew from the east, the other as the turn of the west wind came. The cellular party apparently triumphed, but it has silently and mysteriously disappeared, so that we may ask with Hans Breitman: "Where is dad barty now?"

In his paper, Tait refers to a contribution of Dr. Ieslie Roberts in a recent number of the British Medical Journal on the pathology of vegetable hair parasites, and remarks that it interested him greatly because it was a phase of the dermatologic investigation getting as near the laboratory flasks as seems pos. sible. Tait makes a note of the lack of unity of opinion on the part of dermatolngists, with reference to the pathology of vegetable hair parasites. He suggests that from a bacteriologic standpoint they are no further advanced than botanists were in the times of Gussieu and Linnæus, and that the philosophical revolution affected by Darwin has not yet reached them, and refers sarcastically to the amount of drivel that is made to center around the culture tube. $\mathrm{He}$ very tersely says: "What should we say if an investigator provided to classify the trees in an orchard by the shape of their branches or the color displayed by their fruit?" He says logic can not change with the size of the objects or with the apparatus and manipulation. He decries the rapidly appearing successive memoirs which are self-contradictory and which are objectionable and unusual in scientific research, and suggests to us all the necessity of reserving judgment. Indeed he says, many of the opinions maintained so strongly regarding bacteria have passed into medical currency with very little inquiry on the part of those who have reached them. He says their work is carried on without regard to the first principles of Bacon and with an utter disregard to the teachings and examples of Darwin. "The very fundamental thoughts of the evolutionary school of biology are brushed aside, and the great text-book of Sachs might as well have never been written."

Tait criticises the application of Pasteur's law which he so wonderfully illustrated in the process of brewing and wine fermentation, which were of such enormous financial value to the interests involved, as not being applicable to the human body, and says that Pasteur was responsible for leading wildly astray many young doctors who had never studied Bacon and were incapabie of grasping the fact that while the former might be wholly right. in the brewing vat, it required a re-arrangement of the syllogisms to carry the argument to the human body. For instance, take ten thousand human bodies all perfectly alike, as far as we can see, and drop your germs of typhus about and see what will happen. The first remarkable fact to be found is that if the bodies are in Birmingham there will probably be no effect atall. If they are in Edinburgh, no effect, or very little will be seen in the new town; whereas in the old town some commotion will result and there will be about a hundred cases of typhus. These will be distributed in various centers with mathematical proportion, and probably no two cases will be exactly alike.
Clearly then, according to Bacon, the provocative cause lies not in the germs.

We can not ignore some of the bacteriologic perplexities which confront us, however loyal we may be to the germ theory.

1. Pneumococci may exist in the air passage without producing pneumonia.

2. The bacilli coli (which are constantly at home in large numbers in the large bowel) are believed by some to beessentially the same as the typhoid fever bacillus of Eberth and to develop the same biologic and pathogenic characteristics when the soil is favorable. Indeed it is freely stated that the Klebs-Loffler bacillus is often found among the many scores of bacteria infecting apparently healthy mouths.

In a recent paper Bleyer says: "To-day we know with almost absolute certainty that there are two forms of diphtheria, which to the eye of the clinical observer present similar changes in the mucous membrane, inasmuch as both are characterized by the appearance of a pseudo-membrane, at times dirty white, often tinted greenish or yellow white in color. Viewed at the bedside alone, in both you will observe alike, prostration, a febrile condition, swelling of the submaxillary glands, disturbance of respiration often slight it is true, so that a differential diagnosis, clinically, is a qualification to which few of us can lav claim. Notwithstanding this, the two are totally dissimilar. Examined bacteriologically, the exudate of one will reveal nests of bacilli which are found only in the exudate of true diphtheria, while in the other there are no bacilli, but instead the so-called staphylo- and streptococci. The first variety is highly fatal, statistics showing a mortality of almost 50 per cent., while the second, so far as danger to life is concerned, is comparatively harmless." In this latter statement he is wrong, for even these causes may produce fatal blood poisoning.

Skigo reports several cases which he met with during an epidemic of diphtheria where the tonsils were covered with disseminated spots of exudate the size of a pea, and he tells us that there was absolutely no clinical indication by which he could differentiate the cases which ran the violent course of true diphtheria, and those which took the milder course.

Morton examined 200 cases of diphtheria that had been diagnosed by that eminent clinician, Simon of Paris, and found in only 137 of these the Klebs-Löfler bacilli; 43 of the remaining 63 cases proved to be tonsillitis, 13 proved to be croup of which 12 got well without operation, 7 cases proved to be diphtheria with croup, 6 of which developed the bacilli after the operation of tracheotomy had been performed and which Morton says had been due to hospital infection.

In recent investigations made by Park, in the New York Orthopedic Hospital, where the children seem specially liable to attacks of follicular tonsillitis and enlarged tonsils, culture experiments were made and in some instances pure cultures of the typical KlebsLöfler bacillus were found in fair abundance and in others they were scanty. Though the soil was favorable, the bacilli found were evidently not of a virulent character, and from these reports, Chappell, in the New York Medical Record, observes that until these causes were examined, he had supposed that Klebs-Löffler bacillus found in a sore throat meant diphtheria, whether the clinical history and evidence supported that diagnosis or not. He concludes that 
at present writing it seems there are three possibilities :

1. Clinical phenomena of diphtheria without KlebsLöffler bacilli.

2. Clinical phenomena of diphtheria with KlebsLöffler bacilli.

3. Kelbs.Löffler bacilli without any clinical phenomena of diphtheria.

If it be true that diseases attended with the formation of the pseudo-membrane of the throat due to other causes than the Klebs-Löffler bacillus are not followed by the well-known sequelæ of diphtheria, then the term diphtheria, should not be applied to them. The term, diphtheria, has in the past been freely applied to this form, but $I$ think it is a misnomer. The name diphtheroid, which was first applied I believe by Dr. Lester Hall of Kansas City, is much more appropriate, as it expresses the thought that the disease is like diphtheria.

It is said that there is no germ that is so variable in its virulence as the Klebs-Löffler bacillus, (but all forms of life vary in their degree of lustiness) and this may account to some extent for the difference in severity in cases of diphtheria; we are all aware of the fact that there is a great variation in the virulence of all epidemics; whether it be due to atmospheric or other causes, has not been fully established. Another element of confusion to the practical physician is the statement that the pseudo-bacillus found in diphtheria does not vary in any way microscopically from the true Klebs-Löfler bacillus. If this bacillue and others may vary so materially in their lustiness, in their ability to make their way in the world from time to time, may they not also vary in their general appearances to a like degree, and thus create confusion as regards microscopic diagnosis?

In a recent discussion, Dr. C. H. Johnston, of Grand Rapids, Mich., says he can see no good reason for considering these so-called pseudo-basilli as other than true diphtheritic germs, which if planted upon suitable soil may give rise to the most severe forms of diphtheria.

The many conflicting facts presenting themselves to us, may, if they do not prompt us to ask ourselves pathologically, "where are we at?" at least make us hesitate in insisting upon the making of a diagnosis based only upon microscopic demonstrations. Indeed, in spite of the brilliant light which has been thrown upon the subject from the intellectual lamps of many of the best men of the profession the world over, there is reason for our not being entirely absorbed in our study of diphtheria as well as other diseases, by the microscopic and bacteriologic evidence to the exclusion of the bedside clinical evidence.

While personally I am disposed to accept the germ theory, particularly as applied to the infectious diseases, and probably to far more than have yet been discovered, yet I still believe most firmly that there are many diseased conditions dependent upon other things than germs and that clinical facts are stubborn things.

Every true physician never ceases to be a medical student, and he needs to guard himself against too readily accepting the dictum of those whom he may recognize as the masters of the profession. The views of Lawson Tait and the many other able workers of the medical world are worthy of consideration, worthy of being weighed in the balance. I have great respect for the opinion of any man who has given evidence of being an earnest, honest student in science, no matter what his views may be. Our own Dr. Wm. Johnston, one of the Nestors of the medical profession of St. Louis, one of the most indefatigable delvers in the field of literature, medical and general, when he antagonizes the germ theory of disease, we must remember is in very good company and his views are worthy of our most profound attention. At the same time the younger workers in the scientific vineyard who spend most of their time looking through the small end of a microscope and into the depths of test-tubes, mingling with laboratorial outfits, should have a care lest they develop an intellectual myopia which will preclude their appreciation of the importance of bedside observation, of clinical evidence. They should each and every one remember that bacteriology is not all of the science of medicine, and that the bulk of medicine is art rather than science, and other things being equal, the careful student of human nature, the close observer and the energetic worker in the sick room, after years of experience, is often far more competent to make a diagnosis of a given disease by clinical evidence alone than they, with their microscopes and culture tubes, without such knowledge and experience. Who of us who have listened to the able and forceful words of a venerable teacher of surgery in St. Louis, his teachings based upon knowledge gained from reading not only books but the symptoms of disease, would not as soon accept his dictum with reference to a tumor, its benignancy or malignancy, its necessity for removal, as the most expert microscopic opinion taken alone.

Any physician of experience worthy of the name, can make a diagnosis of pulmonary tuberculosis on the clinical evidence alone, and bring the microscope to his aid only for confirmatory purposes. But the true physician is ever receptive and unprejudiced whether in the sick-room or the laboratory-he who knows it all generally knows very little.

Like syphilis, diphtheria is probably primarily local, as it can be inoculated upon a denuded surface, but like syphilis, general in its effects, it so promptly affects the system at large that the removal of the infected point by the cauterization of the membrane in the one case or the excision of the primary chancre in the other, will not prevent constitutional systemic poisoning. Time was, when in an empirical way, physicians immediately attacked these exudates in the air passages with violent caustics, of ten doing greater injury to the surrounding parts than to the invading membrane.

As syphilis has been in modern times greatly modified and its asperities softened by the recognition of the importance of local cleanliness and the securement of favorable constitutional conditions, so I believe may diphtheria. A suitable soil with depressed constitutional conditions, in other words a dirty suppurating neglected angina (as in scarlet fever, acute tonsillitis or quinsy) with perverted secretions throughout the alimentary canal, an already existing blood poisoning produced by the numerous streptococci and staphylococci present in the faucial field, presents conditions favorable to the development of the most brutal and lusty Klebs-Löffer bacilli imaginable.

One should hesitate long before relinquishing many of the valuable measures which have stood the test of time, and accepting the newer therapeutic sugges- 
tions, even including that of Behring, for he undoubtedly originated and developed the antitoxin treatment of diphtheria. Roux, who is a skilled bacteriologist, and presented remarkable results in a definite manner at the International Congress of Hygiene at BudaPesth last summer, freely accords the credit of this discovery to Behring. The literature that has been presented during the past few months in favor of the blood serum therapy as applied to diphtheria, is enormous, and no candid careful observer can ignore the fact that the great bulk of it is favorable.

Nearly a hundrea years ago, Edward Jenner presented to the world the original thought in the direction of treating disease in man by a product from a lower animal which had previously been acted upon by the same disease. In other words, Jenner's application of vaccination for smallpox was the first move in the direction of the blood serum therapy, for we have no reason to doubt that vaccinia on the udder of the cow is probably primarily the result of smallpox introduced into the same animal. It is true that the workers in this field are now moving along scien. tific lines and not empirically as did Jenner, and yet he stands an example to all scientists whether they be bacteriologists or bedside physicians, illustrating the thought that in the study of symptoms, the grasping of facts, the application of them, clinical work is after all one of the best means of demonstration in the hands of the physician. It will be remembered, too, that Jenner did not publish to the world his work until he had elaborated it, perfected it, and extended it over a period of twenty-five years.

The best report of the antitoxin treatment of diphtheria has been through the Unitea States Marine. Hospital Service. 'Surgeon-General Walter Wyman huving delegated Surgeon J. J. Kinyoun, a skilled bacteriologist, to visit the Pasteur Institute and study thoroughly the work of Prof. E. M. Roux, he presents his official report in the weekly abstract published by the Marine-Hospital Service under date of Oct. 20, 1894 , and it is indeed the most succinct and reliable article upon this subject that has yet appeared. Too great credit can not be given Surgeon-General Wyman and his subordinate, Dr. Kinyoun, for the report. The essence of the conclusions made by Dr. Kinyoun are contained in the following: "The results obtained by Professor Roux in the treatment of cases of diphtheria are so astounding, that one is almost compelled to ask one's self, 'Is this possible?' but when the methods are known and the array of statistics are given, there can hardly remain a trace of doubt. The future possibilities in this direction can not be cver-estimated as we have in the serum the almust absolute preventive of epidemics of diphtheria."

The great Virchow was for a time opposed to this method, but in a recent meeting of the Berlin Medical Society, he publicly announced that he had to vield to the brute force of unanswerable facts. A full opportunity was furnished for the comparison of results in a large number of cases in Berlin, when treated with and without the blood serum. . Hausman, Virchow's assistant, still opposes the blood serum, but as yet there has appeared no presentment of facts upon which to base his opposition; at least it has escaped my observation. The reports from London, France, Berlin and upon this side of the water, are in the affirmative. The government of France has given to Roux its highest honors in recognition of his work.
And now what shall be said of the present general application of the blood serum therapy? First of all, the remedy is enormously expensive. The animal best adapted for its development is the horse, and the time required is not less than six months. This practically places it beyond the reach of the great masses. If the serum treatment is to become general, then comes the important question, and it is a difficult one, what arrangements are to be made for protecting the community under sufficient and proper guarantee with the material for this treatment? Surely the same arguments would apply in favor of this prophylactic measure (for such it is, as well as a remedial agent) being placed under the control of local boards of health, the same as in vaccination for smallpox. It is to be supposed that health boards have definite knowledge and supervision of the vaccine farms which furnish vaccine virus to the respective communities, and they should have similar supervision over the laboratories where the blood serum is developed. The health boards should know that properly healthy animals are secured, those of proper age, and that the proper sanitary and antiseptic precautions are exercised in the establishment. Even granting that the blood serum therapy has proved its case, until it is available, and even after, we must not lose sight of other remedies which have after long service proved of value, for there can be no doubt that considering the fact that the antitoxin antagonizes only the Klebs-Iöffler bacillus and not the streptococcus and staphylococcus and other pyogenic microörganisms, we are never safe in ignoring our old therapeutic friends in favor of the exclusive use of the new. Reviewing the amount of confusion that exists even among bacteriologists regarding the definite cause of diphtheria, and the different kinds of diphtheria, and the like confusion and uncertainty pertaining to the blood serum therapy, and our inability to make it generally applicable to mixed infections, we are justified in the exercise of the extremest caution in its application.

Diphtheria poison is tenacious of life and may be transported indefinite distances. The disease is always more or less present in crowded communities, possibly inhabiting permanently the sewerage systems of cities. Laboratorically demonstrated, the Klebs. Löfler bacillus is admitted to be found in seemingly healthy mouths frequently. A large number of the children in every community at certain seasons of the year through the medium of follicular tonsillitis, enlarged tonsil's and catarrhal disturbances of the air passages, and scarlet fever angina, present themselves as standing invitations to diphtheria. A bacteriologic demonstration of true diphtheria requires twenty-four or forty-eight hours. Remembering all these facts, every case of sore throat should be isolated and treated as a suspect. While in no case acting as an alarmist, the physician should be a guarder and a protector of his patients, and should from the beginning inform the family of the possibilities of any sore throat developing into a diphtheria.

The foregoing being true in every cace of sort throat, the treatment from the first should be antagonistic to diphtheria, locally and constitutionally, no matter how benign it may appear, no matter whether the Klebs-Löffler bacillus be suspected or discovered. Every case of sore throat should be treated in a cleanly way and every hygienic rule should be ob. served. The ideal treatment for tonsillitis, the an- 
gina of scarlet fever and la grippe, which may be classed under the head of suspects, is a favorable preliminary treatment for any case of diphtheria, and this is briefly, a prompt purging of the patient, emptying the intestinal canal for future service as a food tract, the administration of local applications which serve as soothers and cleaners of the disturbed mucous membranes and are as nearly germicides as can be made. Under this head comes hydrozone and pyrozone, which are definite strengths of peroxid of hydrogen, and if too irritating may be diluted onehalf. This application should be followed by Lister. ine, Pasteurine or Katharmon, applied preferably with a hard rubber syringe, the nozzle of the same guarded by a soft piece of rubber tubing two or three inches long, to protect the throat from being wounded. The keeping of the rasal passages well open by the means of liquid albolene ( 5 grains of carbolic acid to each ounce) protects the mucous surfaces and renders the infection at these points less probable. The internal administration (from the beginning) of the benzoate of soda, in doses ranging from 5 to 20 grains, well diluted with plain water every one or two hours, according to the age of the patient, serves as a stimulator of secretions and is the best remedy for prompt reduction of a benign inflammation of the tonsils, it being equally valuable in the removal of exudations. A dry engorged condition of the mucous membranes with all secretions presenting a tenacity and a disposition to stick to the surfaces, is soon changed under the saturating doses of the benzoste of soda, into a loosening of all exudations and a moist condition of all the surfaces. A flow of mucous from the parts is stimulated and exosmosis rather than endosmosis is encouraged. Salkowski in 1879 announced that saturated solutions of the benzoate of soda rendered the diphtheria membrane, if bathed in them, absolutely inert, so that the remedy is more or less localiy antiseptic. In addition to the remedy suggested, the bichlorid of mercury should be given from the beginning, starting with $1-50$ of a grain or even 1-32 well diluted, to a child of 2 years, and increasing in proportion to the age, for at least twenty-four hours, the same dose to be continued every two or three hours thereafter as long as the conditions call for it, unless too free a catharsis be produced. Clinical facts deduced by others, as well as those coming under my own observation, are absolutely convincing that the internal use of the bichlorid of mercury is antagonistic to the toxic effect of the diphtheria poison, the same as it is to the Lustgarten germ of syphilis (if there be one) and to all other germs as weil, including even tuberculosis when given discreetly, and it is a local antiseptic.

Our forefathers were not far wrong when they gave calomel and other forms of mercury in reasonable doses to their cases of diphtheria, typhoid fever and cholera; but mercury is not the only remedy which has for centuries been given empirically and yet proved to be scientifically correct. We have been giving opium for two thousand years as a reliever of pain, and yet we do not know how it acts; we only know that it does.

The thoughtful physician should never lose sight in the management of his throat cases, of feeding, and indeed within twenty-four or forty-eight hours, having placed the alimentary canal in the best possible shape for the digestion and assimilation of food, the nutrition, in a form to be readily digested (use it predigested if necessary) and assimilated in proper quantities at proper times should be furnished, for after all, good well fed blood can repel successfully most of the bacilli and their toxins. Stimulation should be ever kept in mind but not applied too soon. When we recall the demoralization of the digestive tract and the nerve centers produced by the excessive use of alcohol in adults, we should have a care in our administration of alcoholic stimulants to delicate children. I have found a pine-apple peptic wine known as Zumo-Anana of great service as a stimulant. Remedies, either local or constitutional, should not be given so frequently or be of so irritating a character as to interfere with the digestive function or with the proper amount of rest on the part of the patient.

To epitomize, then, whether the blood serum therapy be applied or not, our treatment of our diphtheria cases should include a full regard for :

Elimination, the keeping open of the secretory and excretory system of glands.

Disinfection, local and general, of the attacked surfaces, the patient and his environment, should be applied, even though the case be only a suspect and present none of the evidences of the more infectious disease.

Nutrition of a character to be most promptly util. ized, and nothing is better in this direction than peptonized milk, and milk has the advantage in this form of being soothing to the inflamed surfaces of the throat. Ice cream made of good materials is often gratefully received by the patient and is quite digestible.

Stimulation, but not until it is required, for he who crosses his bridges before he gets to them makes a mistake. Oats are better for the thorough.bred roadster than the whip, unless the latter be used upon the animal when in the middle of a bridge which is breaking down; and then the whip serves well to spur the flagging energies, and so with stimulation in disease.

Tranquillization both of body and mind should be the rule from the beginning, and to this end the victim should never be given the diagnosis of his case, and no application, no remedy, nothing should be permitted to interfere with a full amount of sleep within the twenty-four hours. If necessary, sedatives to secure rest should be given and they should be given for an effect. Small doses of a sedative of ten excite, whereas large ones result happily.

Rest is one of the sweetest words in our language, and in the management of no disease is this more true than in diphtheria.

In closing, let me insist upon the thought that in the majority of cases the microscope is only valuable for the confirmation of the diagnosis, though exceptionally it is essential to the making of a diagnosis, and whether we get our own consent to the application of the blood serum therapy or not, let us never lose sight of the proper toilet of the throat, the proper use of our accepted and well tried remedies in addition.

The child with sore throat, as well as the other members of the family, should be given the benefit of the doubt, and isolated and treated always with the possibilities of diphtheria in mind.

The Forty-sixth Annual Session of the American Medical Association will be held in Baltimore, Md.. on Tuesday, Wednesáay, Thursday and Friday, May 7, 8, 9 and 10, commencing on Tuesday, at 10 A.M. 